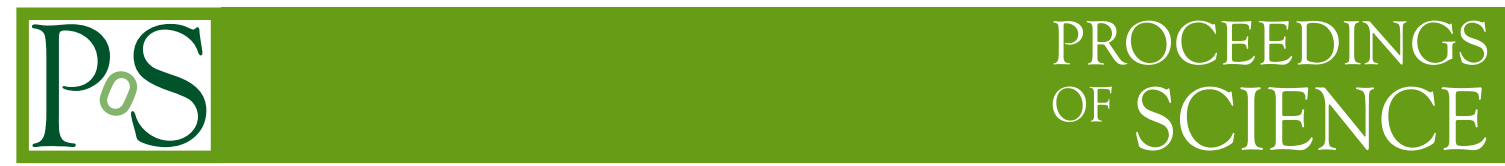

\title{
W Measurement at PHENIX
}

\author{
Chong Kim** \\ University of California, Riverside \\ Department of Physics \& Astronomy, Riverside CA 92521 USA \\ E-mail: ckim.phenix@gmail.com
}

The W measurement at RHIC (Relativistic Heavy Ion Collider) provides unique access to the sea quark polarization of the proton. By measuring decay leptons from parity-violating $\mathrm{W}$ bosons, which only coupled to left-handed quarks and right-handed antiquarks, a clean, fragmentation free measurement is possible in addition to natural flavor separation. In this talk, we present status and results of single longitudinal spin asymmetry $\left(A_{L}\right)$ measurements at PHENIX with data collected in 2011-2013.

XXVI International Workshop on Deep-Inelastic Scattering and Related Subjects (DIS2018)

16-20 April 2018

Kobe, Japan

\footnotetext{
* Speaker.

${ }^{\dagger}$ for the PHENIX Collaboration
} 


\section{Introduction}

\subsection{Motivation}

Since the Spin Crisis in late 1980s, it is well known that the proton spin $\frac{1}{2}$ is not a simple sum of its constituent quarks. According to the Jaffe-Manohar spin sum rule the proton spin $\frac{1}{2}$ can be decomposed into several components as follows:

$$
S_{p}=\frac{1}{2}=\frac{1}{2} \Delta \Sigma+\Delta G+L_{z}
$$

where $\Delta \Sigma$ is the contribution of quark and anti-quark $(\Delta q+\Delta \bar{q})$ spins, $\Delta G$ is the contribution of gluon spins, and $L_{z}$ is the orbital angular momenta originated from previous components, respectively. Inclusively, the $\Delta \Sigma$ is very well defined along Bjorken $\mathrm{x}$ down to $\sim 10^{-3}$ thanks to the DIS and SIDIS results. However, in the manner of flavor separated constraint it's still rather poorly understood, due to the fixed target experiment's relatively low energy scales and dependence on the fragmentation functions. The $\mathrm{W}$ measurement at RHIC provides an elegant solution to these fundamental weakness of traditional fixed target experiments.

\subsection{RHIC and PHENIX}

In 2011-2013, the Relativistic Heavy Ion Collider (RHIC) collected a large amount of polarized $\mathrm{p}+\mathrm{p}$ data with longitudinal beam polarization setup in $\sqrt{s}=500$ (2011) and 510 (2012-2013) $\mathrm{GeV}$. In the manner of integrated luminosity they exceed the previously collected data in 2009 by at least a factor of 20, with improved average beam polarization of $\langle P\rangle>50 \%$.

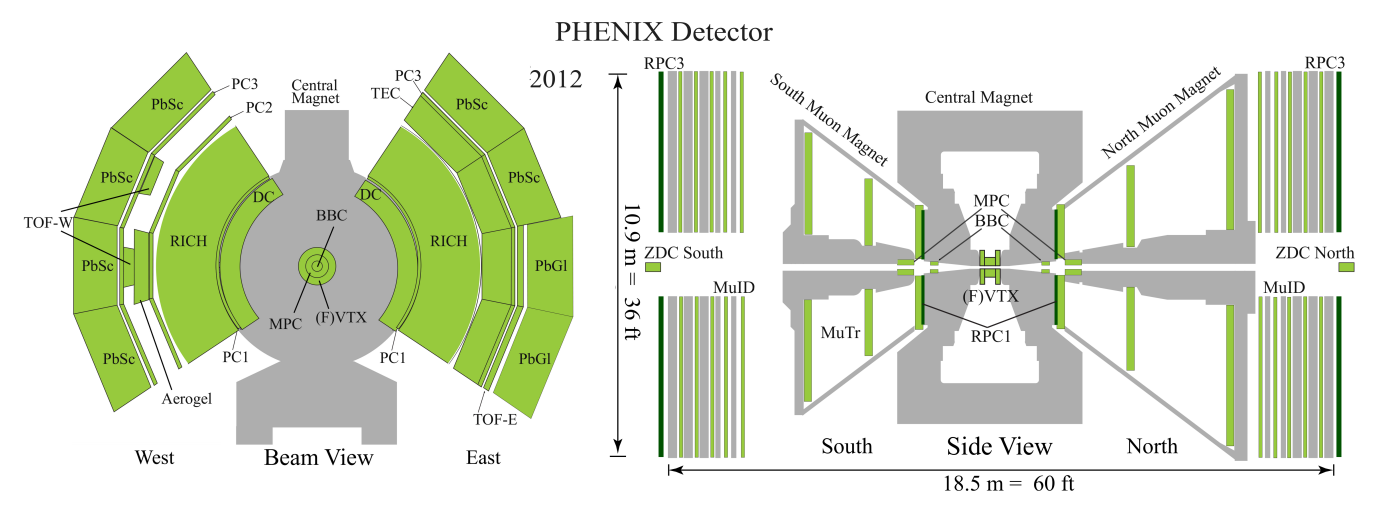

Figure 1: Configuration of PHENIX detectors at 2012

The PHENIX detector is composed of two main parts: the Central Arms in midrapidiry with coverage of $|\eta|<0.35$ and $\Delta \phi=\frac{\pi}{2} \times 2$, and the forward Muon Arms in forward rapidity with coverage of $1.2<|\eta|<2.2(\mathrm{~S}) / 2.4(\mathrm{~N})$ and full azimuth. Note that the W measurement at PHENIX is also separated into two main parts due to these two separated main detector systems. 


\section{W measurement at PHENIX}

\subsection{W measurement at RHIC}

The W measurement at RHIC has a couple of major advantages over traditional SIDIS experiments. First, a W boson only couples with positive helicity anti-quark and negative helicity quark. Therefore by knowing each proton beam's polarization direction and produced W boson's charge its flavor and helicity can be naturally decomposed. Second, RHIC W measurement utilizes W decay leptons as the probe. Since no fragmentation process is included, this measurement is free from any fragmentation functions, which are a major source of uncertainty in the current understanding of anti-quarks' polarization.
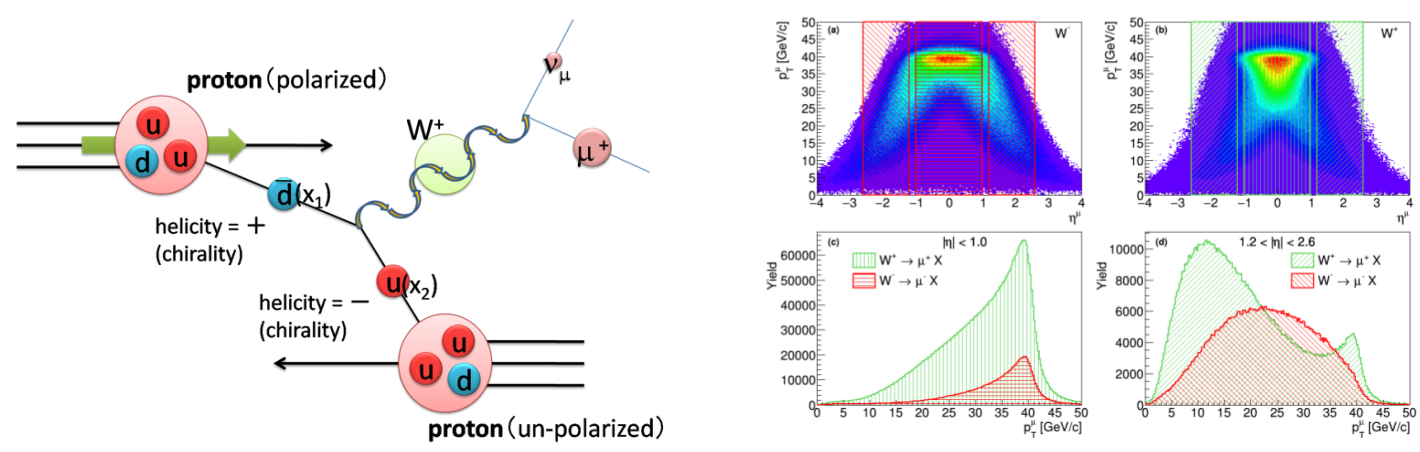

Figure 2: Schematic diagram of W boson production in polarized $\mathrm{p}+\mathrm{p}$ collision (left) and $\mathrm{W}$ decay muons' $p_{T}$ distribution by their charge sign and given pseudorapidity (right) [4]. Note that the right plot is obtained from PYTHIA6 simulation.

The physics observable for the measurement is single longitudinal spin asymmetry $\left(A_{L}\right)$,

$$
A_{L}=\frac{1}{\langle P\rangle} \frac{\sigma_{+}-\sigma_{-}}{\sigma_{+}+\sigma_{-}}=\frac{1}{\langle P\rangle} \frac{N_{+}-R N_{-}}{N_{+}+R N_{-}}
$$

where $\langle P\rangle$ is average beam polarization, $\sigma_{ \pm}$is longitudinal cross section in positive ( + ) or negative (-) beam helicity, $N_{ \pm}$is number of $\mathrm{W}$ decay leptons collected in given helicity, and $R$ is relative luminosity between each helicity bunches, respectively.

The $\mathrm{W}$ measurement at PHENIX can be separated into two main parts: the $W^{ \pm} \rightarrow e^{ \pm}$at midrapidity and the $W^{ \pm} \rightarrow \mu^{ \pm}$at forward rapidity, respectively. In addition to the different detector configurations, the $\mathrm{W}$ decay leptons' kinematic characteristics in each rapidity region enforces each measurement to take a very different approach. As can be seen in right plot of Figure 2, a distinct Jacobian peak can be seen in midrapidity, however, the peak in forward rapidity is either heavily suppressed or cannot be seen at all. Due to this feature the $W^{ \pm} \rightarrow e^{ \pm}$relies on the Jacobian peak for the signal extraction, while the $W^{ \pm} \rightarrow \mu^{ \pm}$largely relies on W likelihood which defined by multivariate analysis.

Also note that unlike W results at STAR, both parts of PHENIX detectors cannot discriminate $Z^{0}$ bosons from final candidates, thus the final $A_{L}$ results include contamination from $Z^{0}$ bosons as well, although their contribution is relatively small. 


\section{$2.2 W^{ \pm} / Z^{0} \rightarrow e^{ \pm}$at midrapidity}

As explained $W^{ \pm} \rightarrow e^{ \pm}$utilizes the Jacobian peak for the signal extraction. To obtain high purity $\mathrm{W}$ decay electron candidates an isolation cut was defined as follows: $r_{i s o}=\left(\Sigma E_{i}\right) / E_{e}$, where $E_{i}$ is the ith cluster energy of EMCal and track $p_{T}$ around the electron candidate in a cone with $\mathrm{r}=$ 0.4 in $\eta$ and $\phi$ [3]. Though minor fluctuations exist by data taking year and charge, the isolation cut effectively removed about $90 \%$ of the backgrounds while keeping more than $90 \%$ of the signal.
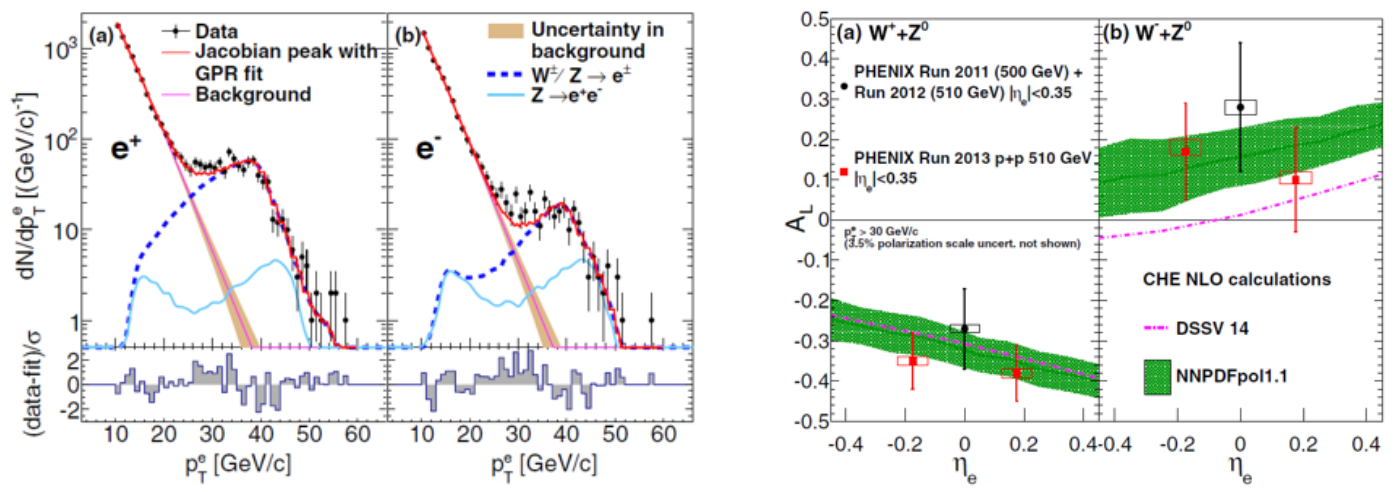

Figure 3: Final $p_{T}$ spectra of $W^{ \pm} / Z^{0} \rightarrow e^{ \pm}$at $\sqrt{s}=510(\mathrm{GeV})$ from 2013 (left) and Final $A_{L}$ results from 2011-2013 (right).

Once high purity candidates are obtained, the backgrounds in signal dominant region $(30<$ $p_{T}<50 \mathrm{GeV}$ ) was estimated by extrapolating backgrounds in the background dominated region $\left(10<p_{T}<22 \mathrm{GeV}\right)$ by using a Gaussian Process Regression technique.

Right plot of Figure 3 shows final results of $W^{ \pm} / Z^{0} \rightarrow e^{ \pm}$at $\sqrt{s}=500$ (2011) and 510 (20122013) $\mathrm{GeV}$, with total integrated luminosity of $240 \mathrm{pb}^{-1}$. The fraction of $Z^{0}$ bosons in the signal region was estimated to be $7 \%\left(e^{+}\right)$and $25 \%\left(e^{-}\right)$. The final $A_{L}$ of $W^{+}$shows good match to the theoretical expectation, however, $W^{-}$tends to have larger asymmetry than DSSV14 [2], which was also observed at STAR $W^{ \pm} \rightarrow e^{ \pm}$in 2011-2012, as well.

\section{$2.3 W^{ \pm} / Z^{0} \rightarrow \mu^{ \pm}$at forward rapidity}

The analysis of $W^{ \pm} \rightarrow \mu^{ \pm}$has a lot more challenges in the manner of signal extraction compared to the previous $W^{ \pm} \rightarrow e^{ \pm}$. First, as explained already the Jacobian peak is seriously suppressed. Second, PHENIX muon arm is non-hermetic, thus a few traditional technique such as isolation cannot be used. Third, both muonic and hadronic backgrounds are dominant in all region of interest. Fourth and Finally, due to the limited resolution of main tracker (MuTr) there is serious smearing in the reconstructed $p_{T}$.

According to the detailed simulation study based on PYTHIA6 and NLO calculations, most of backgrounds (both muonic and hadronic) can be effectively suppressed with high enough $p_{T}$ cut. However, large amount of backgrounds combined with $p_{T}$ smearing in the reconstruction degrades rejection power of $p_{T}$ cut greatly. As a result, any remnant of Jacobian peak is completely washed out in addition to overwhelming backgrounds induced in all region of interest. 

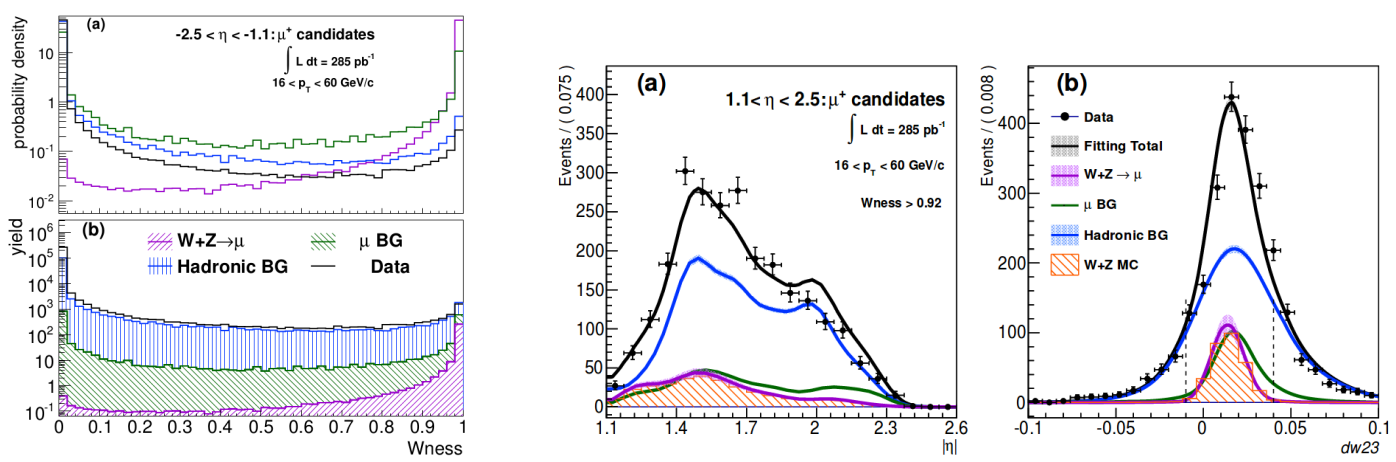

Figure 4: Wness distribution of each type of muon candidate (left) and Results of 2D fit on final muon candidates (right).

Due to the problems previously explained, the backgrounds among muon candidates cannot be simply rejected by applying a single kinematic variable or two. Therefore multiple kinematic variables were applied in the analysis and a likelihood to the $\mathrm{W}$ signal, Wness, based on those variables were defined to control them effectively. The definition of Wness is as follows.

$$
\text { Wness }=\frac{\lambda_{\text {sig }}}{\lambda_{\text {sig }}+\lambda_{b g}}
$$

Where $\lambda$ indicates a combined probability distribution based on either W MC $\left(\lambda_{\text {sig }}\right)$ or data $\left(\lambda_{b g}\right)$. Especially the latter is effectively a background due to its low signal fraction $(<0.1 \%)$.

By applying high Wness in general higher $\mathrm{W}$ signal purity can be expected among muon candidates, however, applying tighter Wness cut also means less statistics will be available as trade off. After detailed optimization study, the Wness $>0.92$ was applied for the selection of final muon candidates. Then yields of $\mathrm{W}$ signals among final candidates were estimated by using $2 \mathrm{D}$ unbinned maximum likelihood fit. The resulting signal to backgrounds ratio were vary from $15 \%$ to $28 \%$, depending on charge sign and detector location.
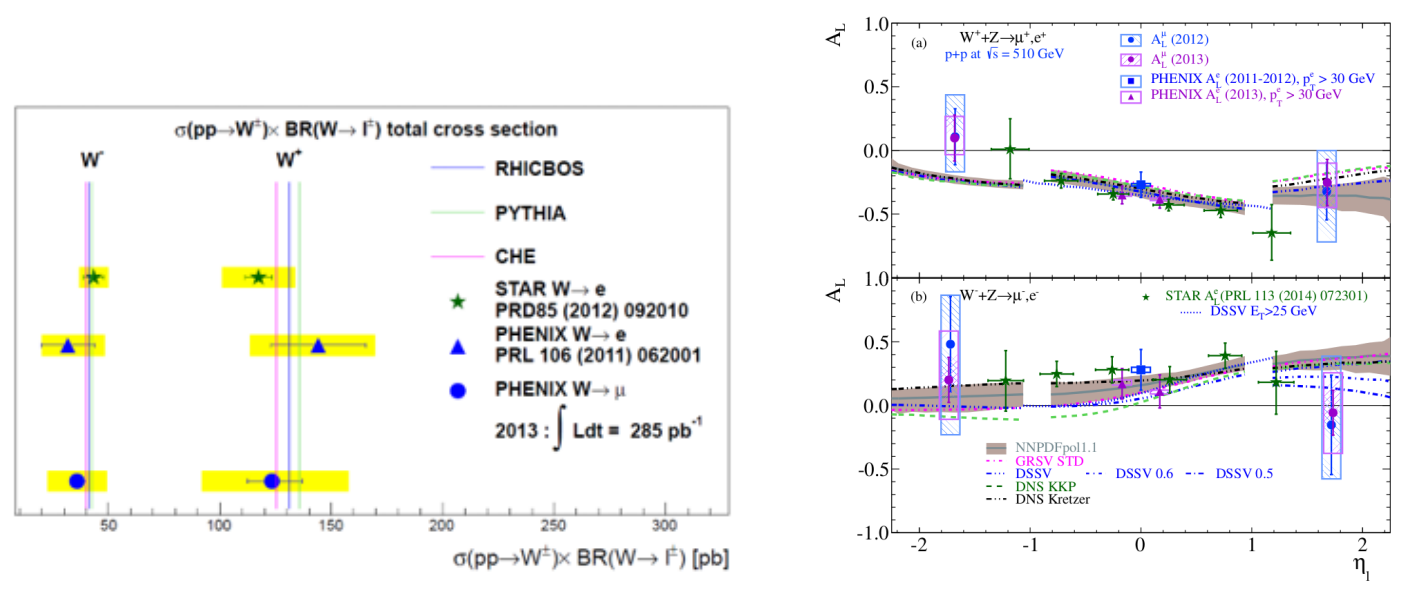

Figure 5: $W^{ \pm} \rightarrow \mu^{ \pm}$cross section in 2013 presented with previous measurements (left), and final $A_{L}$ results from 2012-2013 drawn with previous results in PHENIX and STAR (right). 
The right plot of Figure 5 shows final $A_{L}$ results of $W^{ \pm} \rightarrow \mu^{ \pm}$at $\sqrt{s}=510 \mathrm{GeV}$ in 2012-2013, with total integrated luminosity of 53 (2012) and $285(2013) \mathrm{pb}^{-1}$. The fraction of $Z^{0}$ bosons in final candidates was estimated to be $18 \%\left(\mu^{+}\right)$and $22 \%\left(\mu^{-}\right)$. The final $A_{L}$ show reasonable match to the theory for forward $W^{+}$and backward $W^{-}$, however, there exist some deviation in backward $W^{+}$and forward $W^{-}$.

For the discrepancy in backward $W^{+}$, unlike the $W^{-}$, the production of $W^{+}$in forward rapidity is always a mixture of up and anti-down quark from either proton which contributes at a different $\mathrm{x}$. Therefore this discrepancy could be originated from unpolarized anti-down at higher x. For the discrepancy in forward $W^{-}$, although the exact reason is unknown, the result seems favor a DSSV scenario which d-quark polarization changes sign and becomes positive at very high $\mathrm{x}(\mathrm{x}>0.5)$.

\section{Summary}

PHENIX has measured single longitudinal single spin asymmetries $\left(A_{L}\right)$ of $\mathrm{W}$ decay leptons in different pseudorapidity. The $W^{ \pm} \rightarrow e^{ \pm}$at midrapidity from 2011-2013 shows good match to the existing results at STAR, including the discrepancy to the DSSV14 of $W^{-}$. The $W^{ \pm} \rightarrow \mu^{ \pm}$ at forward rapidity in 2012-2013 shows some slight deviations from the theoretical expectations, However, please note that this is the first $\mathrm{W}$ measurement at $|\eta|>1$.

\section{References}

[1] D. de Florian, R. Sassot, M. Stratmann, and W. Vogelsang, Phys. Rev. D 80, 034030 (2009)

[2] D. de Florian, R. Sassot, M. Stratmann, and W. Vogelsang, Phys. Rev. Lett 113, 012001 (2014)

[3] A. Adare et al. [PHENIX Collaboration], Phys. Rev. D 93, 051103 (2016)

[4] A. Adare et al, [PHENIX Collaboration], arXiv:1804.04181 (2018) 